\title{
A microcomputer-dependent statistics and design course
}

\author{
RICHARD S. LEHMAN \\ Franklin \& Marshall College, Lancaster, Pennsylvania
}

\begin{abstract}
A one-semester course in statistics and research design is described. All computations are carried out by a Macintosh computer. No hand calculation is expected of the students, and little class time is spent on computational procedure. The course covers much more statistical content than is the norm for a one-semester course, and student understanding of the logic of the material and its application is enhanced.
\end{abstract}

At Franklin \& Marshall, psychology is classified as a natural science, and its program includes extensive handson research experience. The required experimental design and statistics course described here is a prerequisite for most of the intermediate courses (including such laboratory courses as psychobiology, cognitive processes, and learning, sensation, and perception) and for all eight of the advanced research seminars (e.g., infant behavior, human perception and action, environmental psychology, and biological psychology), of which each major must complete three. These intermediate and advanced courses place heavy demands on the design and statistics course. Students are expected to have a full introduction to descriptive and inferential statistics through at least twofactor ANOVA with repeated measures and follow-up tests, bivariate linear regression and correlation, and good selection of library skills, not to mention the ability to write research proposals and reports in correct APA (American Psychological Association) style.

The computing environment at Franklin \& Marshall is conducive to developing a computer-dependent sophomore-junior course that can accomplish the needs outlined here. About 70\%-80\% of incoming students purchase Apple Macintosh computers. There are Macs in all faculty and administrative offices, and there is ready access to them at several sites on campus for the $50 \%$ of the students who do not own their own. In addition, the psychology department has equipped its introductory/statistics laboratory suite with 16 512K Macintoshes, which allows the experimental design and statistics course to have a weekly laboratory session with one or two students per computer.

The general strategy is to remove all computational burden from the students. Ordinary statistics courses spend a lot of time teaching computation and consequently do not have enough time to cover much about the logic of the research methods that use the statistics, about how to select and use the right statistic, about how to interpret the results of a statistical analysis, or about how to describe

Please address all correspondence to the author at: Department of Psychology, Franklin \& Marshall College, P.O. Box 3003, Lancaster, PA 17604. research results in proper fashion. These are the really important considerations for a statistics course; obtaining a numerically correct answer is also important, but it can often be an impediment to fully understanding what is really going on.

Some instructors may complain that eliminating hand computation will remove an important element of "getting the feel for the data" from the student's education. I argue against that: To the contrary, freeing the student from the drudgery of calculation allows more time for thorough exploration of the data. Modern computer packages make recalculation so simple that it is very easy to suggest that students redo their analyses in many different ways, each way leading to perhaps a better understanding of the data. For example, how many instructors would even consider presenting their students with a 100 -case data set on two variables and require that the data be scatterplotted in normal, semi-log, and $\log$-log forms and that correlation and regression statistics be calculated, both before and after square-root and log transformations on both of the variables? This would be unthinkable with hand plotting and computing but would be a short few minutes' work with an appropriate software package. Or how many would feel free to ask their classes to generate hundreds of samples from a specified population and compute confidence intervals to illustrate many times that the interval actually contains the population mean? Or how many could have their class generate empirical power functions by counting how many Type II errors were actually made in $\mathbf{3 0 0}$ simulated experiments?

\section{SYLLABUS}

The course meets for three 50-min lecture periods and a 3-h laboratory session each week. Table 1 presents a brief outline for the course, showing the approximate lecture and laboratory topics by week. The students read a research methods text (Elmes, Kantowitz, \& Roediger, $1985)$ along with the lecture presentation. By and large, the text covers research methods, and the lecture presents statistical topics. Occasionally, but not usually, the lecture ties directly to the reading, and no particular effort 
Table 1

Topic Outline

\begin{tabular}{rll}
\hline Week & \multicolumn{1}{c}{ Topic } & \multicolumn{1}{c}{ Lab } \\
\hline 1 & Research methods & Introduction to The Data Desk \\
2 & Logic and methods & Library skills \\
3 & Descriptive techniques & Descriptives and graphing \\
4 & Descriptive techniques & Descriptive statistics/correlation \\
5 & Descriptive techniques & Normal distributions/sampling/transformation \\
6 & Inference & Sampling distributions \\
7 & One-group research & Confidence intervals/Z and $t$ tests \\
8 & Two-group research & Two-sample $t /$ power functions \\
9 & Multiple-group research & Nonparametrics/regression \\
10 & Multiple-group research & ANOVA/data editing and preparation \\
11 & Two-factor designs & Post hoc tests /two-way ANOVA \\
12 & Two-factor designs & Two-way post hoc tests/plotting \\
13 & Two-factor designs & Within-subjects ANOVA \\
\hline
\end{tabular}

is made to coordinate the two, other than assuring that research design topics are read about in the same order as the lectures that concern the appropriate analytic techniques. Although Elmes et al. (1985) has some statistical material in an appendix, it is mostly a "cookbook" that assumes hand computations. No other statistics text is required, and students with questions beyond the short conference level are referred to "any standard stat book" for more detail. In the future, some text that presents logic and not much computation would be a reasonable addition to the course, because it would give the students a source for future reference, if nothing else.

Two data analysis programs (The Data Desk [Velleman \& Lefkowitz, 1985] and CLR ANOVA [Clear Lake Research, 1986] are taught and used in the laboratory sessions. Those two programs (along with Cricket Graph) are used regularly to illustrate both lecture and laboratory presentations.

\section{ASSIGNMENTS AND EXERCISES}

There are two forms of assignments in the courseweekly lab exercises, and designs and analyses. The weekly exercises follow from the lecture and laboratory topics of the week and include various analysis assignments, graphing exercises, and simulation studies using The Data Desk's data-generation facilities. Weekly exercises always include at least one problem in which the students are required to analyze a set of data and present the results as a complete APA-style results (or combined results/discussion) section. This assignment forces writing, thinking, and communications into what otherwise might be merely a computer assignment.

During the semester, the students also complete two sets of designs and analyses. The design is basically an APAformat introduction and methods section. This "proposed experiment" is graded and returned along with computergenerated data. Then the students conduct the necessary analysis and submit it with interpretation as APA-format results and discussion sections. Students are allowed to select topics for their simulated research that are of interest to each individual, although the form of the design (e.g., two-group design, one-way ANOVA, etc.) is usually specified. Designs and analyses are marked for both writing and content and must adhere to APA style requirements. Students are encouraged to do all of their writing and to prepare their tables and illustrations on the computer.

\section{LABORATORY SESSIONS}

Table 1 shows the weekly laboratory topics. Each session includes a discussion of the just-completed exercise and covers some new computer material that relates to the lecture topic of the week; typically, it has an in-class data-collection (or generation) exercise and an on-line presentation and interpretation of the data. (The example experiments dealing with transformations and correlation, confidence intervals, and power functions were done as in-laboratory exercises this semester.) Each laboratory session ends with a period of time for students to work on their weekly assignment with the instructor and a teaching assistant available for help and explanation. Some students opt to leave and do the computer work at home.

\section{OUTCOMES}

Outcomes have been investigated both formally and informally, as is normally the case with most instructional innovations. On the informal level, I can report that student satisfaction is high, as is carry-over into the intermediate and advanced courses that require a thorough understanding of the material. In addition, the expanded coverage, particularly of the ANOVA topics, has made it possible for instructors in other courses to add new material, since they no longer have to teach, as was sometimes the case, a short course on some particular ANOVA design.

Since the new course covers material that could not be covered under the old curriculum, old final examinations could not be used to test mastery in the new course. However, analysis of some of the questions that were common during different semesters clearly shows that, in the new course, students are at least as able to understand, interpret, and evaluate research data and designs as they were under the old syllabus. And since they now have more design and analysis techniques available, the 
course represents progress in content coverage with no decline in understanding.

Pre- and postcourse surveys indicate that students gained considerable appreciation for the nature of research and, where statistics fit into the "big picture," greatly increased confidence in their computer abilities, and considerable satisfaction with what they had learned.

\section{REFERENCES}

Clear Lake Research. (1986). CLR ANOVA: Analysis of variance program for the Apple Macintosh. Houston: Author.

Elmes, D. G., KaNTOWTTZ, B. H., \& RoEDIGER, H. L. (1985). Research methods in psychology (2nd ed.). St. Paul, MN: West.

Velleman, P. F., \& Lefkowitz, J. M. (1985). The Data Desk handbook. Ithaca, NY: Data Desk. 